\title{
A New Dynamic Multicriteria Decision-Making Approach for Green Supplier Selection in Construction Projects under Time Sequence
}

\author{
Shi Yin, Baizhou Li, Hengmin Dong, and Zeyu Xing \\ School of Economics and Management, Harbin Engineering University, Harbin, Heilongjiang Province 150001, China \\ Correspondence should be addressed to Baizhou Li; acadch@hrbeu.edu.cn
}

Received 20 October 2017; Accepted 5 December 2017; Published 26 December 2017

Academic Editor: Konstantinos Karamanos

Copyright (C) 2017 Shi Yin et al. This is an open access article distributed under the Creative Commons Attribution License, which permits unrestricted use, distribution, and reproduction in any medium, provided the original work is properly cited.

\begin{abstract}
Nowadays, due to the lack of natural resources and environment problems which have been appearing increasingly, green building is more and more involved in the construction industry. The evaluation and selection of green supplier are a significant part of the development of green building. In this paper, we propose a new dynamic multicriteria decision-making approach in construction projects under time sequence to deal with these problems. First, the paper establishes 4 main criteria and 17 subcriteria for green supplier selection in construction projects. Then, a method considering interaction between criteria and the influence of constructors subjective preference and objective criteria information is proposed. It uses the interval-valued intuitionistic fuzzy geometric weighted Heronian means (IVIFGWHM) operator and multitarget nonlinear programming model to calculate the comprehensive evaluation results of potential green suppliers. The proposed method is much easier for constructors to select green supplier and make the localization of green supplier more practical and accurate in construction projects. Finally, a case study about a green building project is given to verify practicality and effectiveness of the proposed approach.
\end{abstract}

\section{Introduction}

Green building has been a popular research topic from academic and industrial sectors in recent years due to global warming and climate change, which are mainly caused by greenhouse gas (GHG) emissions. According to Yudelson [1], globally, buildings play a central part in causing GHG emissions and they are responsible for more than $40 \%$ of total GHG emissions. Some research institutions conducted an interesting survey which also reported that buildings account for nearly $70 \%$ of GHG emissions in Hong Kong and up to $40 \%$ of total energy consumption. These facts show that green building would play an important role in reducing the amount of GHG emissions emanating from the construction industry. Many countries have launched a series of measures to reduce GHG emissions in the construction industry [2]. To cope with pressure, it is a vital factor for the contractor of construction project to select their suitable green suppliers who have affinity with green ability. Moreover, both qualitative and quantitative factors should be taken into account in the process of green supplier selection as a complex multicriteria decision-making (MCDM) problem [3]. Therefore, it is critically important and necessary to study green supplier selection in construction projects. The research not only provides an effective approach to assist contractors to select their best green suppliers in construction projects, but it is also propitious to cut the costs of the supply chain and achieve GHG emissions reduction and then improve environmental performance of the construction industry.

Research on green supplier selection has widely attracted more and more attention from industries and academia. Many scholars have stressed the importance of selecting suitable criteria in the process of green supplier selection. Lee et al. [4] proposed 5 main criteria for green supplier selection, such as quality, technology capability, pollution control, green products, and green competencies in the high-tech industry. Hsu et al. [5] established 13 criteria of supplier selection in green supply chain management with three main criteria, such as planning, implementation, and management. Kannan and Jabbour [6] and Tsui and Wen [7] thought green supplier 
selection should consider green criteria in environmental aspects, such as waste reduction, green technologies, and use of ecodesign. Gurel et al. [8] established 8 main criteria that include cost, delivery, quality, service, strategic alliance, and pollution control, for green supplier selection. Chen et al. [9] proposed 20 criteria of green supplier selection and evaluation criteria with two dimensions (economic criteria and environmental criteria). Yu et al. [10] took into consideration the economic criteria (price, quantity, and lead time) and environmental criteria (green factors and carbon dioxide emissions) during green supplier selection. Govindan and Sivakumar [11] took into consideration economics (cost), operational factors (quality and delivery), and environmental criteria (recycle capability and GHG emission control). Pang et al. [12] proposed 4 main criteria including use of enterprise low-carbon qualification, low-carbon production and service, low-carbon business operation, and low-carbon innovation, for green supplier selection in low-carbon supply chain. Although these studies exist on the topic of green supplier selection, green supplier selection in construction projects is not specially considered in the above researches. There are some literatures on green supply chain management in construction projects. Zhang et al. [13] identified the strategies that are important for promoting green building technologies adoption in the construction industry. Mokhlesian [14] used the case of three Swedish companies to study supplier selection for green construction projects; the study shows that contractor perception of supplier mastery of green knowledge affects supplier selection. Ruuska et al. [15], Woo et al. [16], and Kim et al. [17] studied the environmental capabilities of suppliers for green supply chain management in construction projects. However, most of them focus on green supply chain management of construction industry and the research on the green supplier selection in construction projects is fairly rare. It is inappropriate for constructors to select green supplier in construction projects. Moreover, comparing with the traditional green supplier selection criteria, such as quality, logistics, and service, constructors must pay special attention to the environmental capabilities, green building technologies, and social factors for green supplier selection criteria in construction projects [18]. This research takes into consideration these aspects, which have been ignored in many studies, as an evaluation criterion to develop a practical green supplier selection model in construction projects.

In recent years, extensive MADM methods have been proposed for supplier selection. Govindan et al. [19] concluded that the most frequently used method is AHP (27.78\%), followed by ANP (16.6\%), DEA (11.1\%), LP (8.76\%), TOPSIS (5.56\%), and multiobjective optimisation (2.77\%). In addition, many methods have been developed to select suitable green supplier based on specific methods that include fuzzy set theory [9, 12, 19-21], genetic algorithm [22-24], structural equation modeling and fuzzy logic [25], and artificial neural network $[26,27]$ and their hybrids. Amid et al. [28] used a weighted max-min model based on AHP to select supplier in a supply green chain. Hsu et al. [5] proposed a carbon management model based on DEMATEL for supplier selection in green supply chain management. $\mathrm{Hu}$ et al. [29] proposed a multicriteria group decision-making method with 2-tuple linguistic assessments for green supplier selection under a fuzzy uncertain information environment. Tavana et al. [30] proposed an artificial neural network (ANN) and adaptive neuro fuzzy inference system (ANFIS) model to assist managers in their supplier evaluation and selection process. Tsui and Wen [7] developed a hybrid multiple criteria group decision-making (MCGDM) method based on AHP, entropy, elimination, and ELECTRE III, and the linear assignment method. Qin et al. [31] developed a new TODIM technique to select green supplier within the context of interval type-2 fuzzy sets. Ghadimi et al. [32] present a multiobjective hybrid fuzzy linear programming model for green supplier selection problem.

However, most of these methods, which do not consider interaction between criteria, can lead to irrational decisionmaking of green supplier selection in construction projects. In fact, there is always an interactive relationship between criteria of green supplier selection, such as complementarity between criteria, the redundancy of criteria, and preference relation of criteria. The Heronian mean (HM) is a mean type aggregation technique, which considers interaction between criteria that makes it very useful in decision-making [33]. Although many scholars proposed HM operator [34], GHM operator [35], and IVIFHM operator [36], there is a lack of further theory and method research into the IVIFSs based on HM operator.

In addition, in real life, past and current information should also be considered when conducting dynamic decision-making of green supplier selection in construction projects, and how to solve the problem of time sequences weight is a key to solving the green supplier selection problem. At present, some of the commonly used time sequence weights are as follows: the arithmetic progression and geometric progression method [37], the binomial distribution method [38], the BUM function method, the normal distribution method [39], the exponential distribution method [40], and the time sequence ideal solution method [41]. However, traditional MADM of green supplier selection problems usually focuses on decision information only in a single period in multicriteria decision-making problems, and even if these weights contain multiple timings, they still may lead to the subjectivity and objectivity in the time sequence weight. Therefore, the above researches still lack a comprehensive methodology with strong practicability to select the green supplier in construction projects.

The main contribution of this paper is developing a new dynamic MADM that considers interaction between criteria under time sequence for green supplier selection in construction projects. To overcome the limitations of these existing criteria of green supplier selection in construction projects, the paper establishes an indicator hierarchy for green supplier evaluation and selection of construction industry by taking into consideration environmental capabilities, green building technologies, and social factors, which can help constructors to identify the potential areas of green suppliers. The proposed method provides a process of dynamic MADM of green supplier selection more comprehensively and accurately in construction projects. In the proposed method, it introduces the interval-valued intuitionistic fuzzy geometric 
weighted Heronian means (IVIFGWHM) operator that considers interaction between criteria. Moreover, in the model, time sequence weight is developed via the multitarget nonlinear programming model that is based on time degree and information entropy. It considers the influence of constructors subjective preference and objective criteria information. The proposed method has been successfully implemented in a case of construction projects to select the best green supplier. Besides, the developed method can be widely used as a structural model for green supplier selection in other industries.

The structure of this paper is organized as follows. Section 2 establishes the criteria for green supplier selection in construction projects. Section 3 draws some related concepts of the proposed approach for green supplier selection. Section 4 proposes a method that considers interaction between criteria under time sequence based on IVIFGWHM operator and time sequence weight for green supplier selection. Section 5 provides a real case study that concerns green supplier selection in construction projects example. In Section 6, we end the paper by summarizing the conclusions.

\section{Green Supplier Selection Criteria}

For most projects, in the construction industry, the client selects the contractor, and then contractor selects suppliers of goods and services. Green Supply Chain Management (GSCM) is very important in the construction industry, a project-based business that may result in environmental pollution. The successful implementation of a project requires selecting green supplier that contributes to the project objective for contractor. GSCM consists of green purchasing, green manufacturing management, green marketing, and reverse logistics [42]. Green supply chain in the construction industry is a functional network structure model, which consists of main parts of construction industry, with building units as the core and logistics, capital flow, information flow, and knowledge flow as the support in the whole life cycle of building projects. In this section, we will introduce the proposed criteria for green supplier selection based on above reviews and the identified criteria mentioned above especially with environmental capabilities, green building technologies, and social factors in the construction industry. We establish 4 main criteria (building materials information, green business operation, potential for sustainable cooperation, and green technology capability) and 17 subcriteria (e.g., building materials price and green and waste materials reclamation) for green supplier selection in construction projects. See Table 1.

2.1. Building Materials Information $C_{1}$. Building materials information is the basic point for green supplier selection in construction projects. In building's construction process, projects demand different product types, such as different types of concrete, steel, and templet, and product structure to guarantee the successful completion of construction projects. Therefore, green supplier selection in construction projects should focus on materials flexibility, efficiency, information, and other aspects of building materials. It is particularly important to provide constructors with high quality and inexpensive building materials or service, such as payment terms, to meet the needs of constructor.

In addition, the green degree of building materials reflects its ability of saving resources and reducing energy consumption, and the higher the green degree is, the more application potential the building materials will have in the future. Meanwhile, it also needs to improve service quality and user experience and strengthen after sales service support. Therefore, building materials information is mainly reflected from four aspects: materials cost, green degree of materials, materials quality, and materials flexibility.

2.2. Green Business Operation $C_{2}$. In the complex and changing market environment, the competitiveness of green supply chain in the construction industry depends on rapid response to the needs of different product types and product structure in building's construction process. High level of green business operation can contribute to reducing carbon emissions which can be reflected by the level of green information sharing, the cost control of transportation, and so forth in the supply chain management of construction industry. In addition, constructors need to consider the financial capability to reduce the risk of cooperation between constructors and their suppliers protection for the successful completion of construction projects. Here, we use level of green information sharing, green logistics, financial capability, and emergency response capability to measure the supplier's green business operation.

2.3. Potential for Sustainable Cooperation $C_{3}$. In the construction industry, the main purpose of establishing green supply chain is to establish cooperative alliance of construction industry, which can reduce building materials cost and obtain more income in projects. Cooperation potential is the premise of establishing strategic alliance, and strong cooperation intention and longtime cooperation are the foundation of establishing strategic alliance.

Corporate green culture can promote the implementation of enterprise strategic objectives of sustainable development virtually. If the green culture between partners can not be integrated, it means that it will lead to different values between constructors and suppliers. Then, it may lead to disputes on both sides of the fierce confrontation, and even relationship is broken. In addition, if constructors want to maintain the long-term stability of green supply chain cooperation, they should choose those suppliers who have advanced management and desire of green cooperation for development. We can measure potential for sustainable cooperation from these four aspects: compatibility of green culture, desire of green cooperation, enterprise reputation, and green image.

2.4. Green Technology Capability $C_{4}$. Green technology capability, which is used to evaluate whether the green supplier meets the requirements of green building, is increasingly crucial to successfully implement green building and attain sustainability goals. Green building technologies (GBTs) are incorporated into building design and construction to make the end product sustainable. Green R\&D innovation includes new launch of building materials and GBTs, and there are 
TABLE 1: Criteria for green supplier selection in construction projects.

\begin{tabular}{|c|c|c|}
\hline Criteria & Main criteria & Subcriteria \\
\hline \multirow{17}{*}{ Green supplier selection in construction projects } & \multirow{4}{*}{$C_{1}$ : building materials information } & $C_{11}:$ materials cost \\
\hline & & $C_{12}$ : green degree of materials \\
\hline & & $C_{13}:$ materials quality \\
\hline & & $C_{14}$ : materials flexibility \\
\hline & \multirow{4}{*}{$C_{2}:$ green business operation } & $C_{21}$ : level of green information sharing \\
\hline & & $C_{22}:$ green logistics \\
\hline & & $C_{23}$ : financial capability \\
\hline & & $C_{24}$ : emergency response capability \\
\hline & \multirow{4}{*}{$C_{3}:$ potential for sustainable cooperation } & $C_{31}$ : compatibility of green culture \\
\hline & & $C_{32}:$ desire of green cooperation \\
\hline & & $C_{33}$ : enterprise reputation \\
\hline & & $C_{34}$ : green image \\
\hline & \multirow{5}{*}{$C_{4}$ : green technology capability } & $C_{41}$ : green certifications \\
\hline & & $C_{42}:$ ecodesign of materials \\
\hline & & $C_{43}$ : green production \\
\hline & & $C_{44}:$ waste materials reclamation \\
\hline & & $C_{45}$ : green $\mathrm{R} \& \mathrm{D}$ innovation \\
\hline
\end{tabular}

many different GBTs applicable in the whole process of delivering building projects. Ecodesign of building materials can reduce environmental pollution in the production process and reduce carbon emissions. In addition, green certification reflects the environmental management capability of green supplier. This main criterion includes 5 subcriteria, such as waste materials reclamation and green $R \& D$ innovation.

\section{Preliminaries}

Here, we introduce some basic concepts and terminologies of intuitionistic fuzzy set (IFS), which will be used in the proposed method. Its definition is introduced as follows.

Definition 1 (see [43]). Let $X=\left\{x_{1}, x_{2}, \ldots, x_{n}\right\}$ be a fixed set. An IFS is an object having the form:

$$
A=\left\{\left\langle x_{i}, \mu_{A}\left(x_{i}\right), v_{A}\left(x_{i}\right)\right\rangle \mid x_{i} \in X\right\},
$$

where $\mu_{A}: x \rightarrow[0,1], v_{A}: x \rightarrow[0,1] . \mu_{A}$ defines the degree of membership of the element and $v_{A}$ defines the degree of nonmembership.

With the condition

$$
0 \leq \mu_{A}\left(x_{i}\right)+v_{A}\left(x_{i}\right) \leq 1, \quad \forall x_{i} \in X,
$$

for each IFS $A$ in $X$, let $\pi_{A}\left(x_{i}\right)=1-\mu_{A}\left(x_{i}\right)-v_{A}\left(x_{i}\right)$, which is called the degree of indeterminacy of $x$ to $A$, where $\pi_{A}: x \rightarrow$ $[0,1]$.

Definition 2 (see [43]). Let a set $X$ be a universe of discourse. An interval-valued intuitionistic fuzzy number (IVIFN) $\widetilde{A}$ over $X$ is an object having the form:

$$
\widetilde{A}=\left\{\left\langle x_{i}, \mu_{\widetilde{A}}\left(x_{i}\right), v_{\widetilde{A}}\left(x_{i}\right)\right\rangle \mid x_{i} \in X\right\},
$$

where $\mu_{\widetilde{A}}\left(x_{i}\right)=\left[\mu_{\widetilde{A}}^{L}\left(x_{i}\right), \mu_{\widetilde{A}}^{R}\left(x_{i}\right)\right] \subseteq[0,1]$ and $v_{\widetilde{A}}\left(x_{i}\right)=$ $\left[v_{\widetilde{A}}^{L}\left(x_{i}\right), v_{\widetilde{A}}^{R}\left(x_{i}\right)\right] \subseteq[0,1]$ are intervals, $\mu_{\widetilde{A}}^{L}\left(x_{i}\right)=\inf \mu_{\widetilde{A}}\left(x_{i}\right)$,
$\mu_{\widetilde{A}}^{R}\left(x_{i}\right)=\sup \mu_{\widetilde{A}}\left(x_{i}\right), v_{\widetilde{A}}^{L}\left(x_{i}\right)=\inf v_{\widetilde{A}}\left(x_{i}\right), v_{\widetilde{A}}^{R}\left(x_{i}\right)=\sup v_{\widetilde{A}}\left(x_{i}\right)$, and for every $x \in X$,

$$
\mu_{\widetilde{A}}^{R}\left(x_{i}\right)+v_{\widetilde{A}}^{R}\left(x_{i}\right) \leq 1 .
$$

Let $\pi_{\widetilde{A}}\left(x_{i}\right)=\left[\pi_{\widetilde{A}}^{L}\left(x_{i}\right), \pi_{\widetilde{A}}^{R}\left(x_{i}\right)\right] \subseteq[0,1]$, which is called the degree of indeterminacy, where

$$
\begin{aligned}
& \pi_{\widetilde{A}}^{L}\left(x_{i}\right)=1-\mu_{\widetilde{A}}^{R}\left(x_{i}\right)-v_{\widetilde{A}}^{R}\left(x_{i}\right), \\
& \pi_{\widetilde{A}}^{R}\left(x_{i}\right)=1-\mu_{\widetilde{A}}^{L}\left(x_{i}\right)-v_{\widetilde{A}}^{L}\left(x_{i}\right) .
\end{aligned}
$$

$\mathrm{Xu}$ [44] called the $\tilde{a}=\left\langle\mu_{\tilde{a}}\left(x_{i}\right), v_{\widetilde{a}}\left(x_{i}\right)\right\rangle$ an interval-valued intuitionistic fuzzy number (IVIFN) and denoted the $\widetilde{a}=$ $([a, b],[c, d])$, where $[a, b] \subseteq[0,1],[c, d] \subseteq[0,1]$ and $b+d \leq$ 1.

Definition 3 (see [44]). Let $\tilde{a}=([a, b],[c, d])$, where $\tilde{a}_{1}=$ $\left(\left[a_{1}, b_{1}\right],\left[c_{1}, d_{1}\right]\right)$ and $\tilde{a}_{2}=\left(\left[a_{2}, b_{2}\right],\left[c_{2}, d_{2}\right]\right)$ are two IVIFNs, and $\lambda>0$. The basic operational laws of IVIFNs can be defined as follows:

(1) $\tilde{a}_{1}+\tilde{a}_{2}=\left(\left[a_{1}+a_{2}-a_{1} a_{2}, b_{1}+b_{2}-b_{1} b_{2}\right],\left[c_{1} c_{2}, d_{1} d_{2}\right]\right)$;

(2) $\tilde{a}_{1} \times \tilde{a}_{2}=\left(\left[a_{1} a_{2}, b_{1} b_{2}\right],\left[c_{1}+c_{2}-c_{1} c_{2}, d_{1}+d_{2}-d_{1} d_{2}\right]\right)$;

(3) $\lambda \tilde{a}=\left(\left[1-(1-a)^{\lambda}, 1-(1-b)^{\lambda}\right],\left[c^{\lambda}, d^{\lambda}\right]\right)$;

(4) $\tilde{a}^{\lambda}=\left(\left[a^{\lambda}, b^{\lambda}\right],\left[1-(1-c)^{\lambda}, 1-(1-d)^{\lambda}\right]\right)$.

Definition 4 (see [45]). Let $\tilde{a}=([a, b],[c, d])$ be an IVIFN; the decision function based on the unknown degree $L(\widetilde{a})$ of $\widetilde{a}$ can be defined as follows:

$$
\begin{aligned}
& L(\widetilde{a})=\left[\mu_{\widetilde{A}}^{L}\left(x_{i}\right)+\mu_{\widetilde{A}}^{R}\left(x_{i}\right)-v_{\widetilde{A}}^{R}\left(x_{i}\right) \cdot\left(1-\mu_{\widetilde{A}}^{R}\left(x_{i}\right)\right)\right. \\
& \left.\quad-v_{\widetilde{A}}^{L}\left(x_{i}\right) \cdot\left(1-\mu_{\widetilde{A}}^{L}\left(x_{i}\right)\right)\right] .
\end{aligned}
$$


Definition 5 (see [46]). Let $Q:[0,1] \rightarrow[0,1], Q(0)=$ $0, Q(1)=1$ be a BUM function; the continuous ordered weighted averaging (COWA) operator can be defined as follows:

$$
\begin{aligned}
F_{\mathrm{Q}}(a) & =F_{\mathrm{Q}}\left(\left[a^{-}, a^{+}\right]\right) \\
& =\int_{0}^{1} \frac{\mathrm{d} Q(y)}{\mathrm{d} y}\left(a^{+}-y\left(a^{+}-a^{-}\right)\right) \mathrm{d} y,
\end{aligned}
$$

where $Q(y)$ is a monotonic function, $a=\left[a^{-}, a^{+}\right] \in \Omega, F$ : $\Omega \rightarrow R^{+}$.

Let $\dot{\lambda}=\int_{0}^{1} \mathrm{Q}(y) \mathrm{d} y$ be an attitude parameter; COWA operator can be defined as follows:

$$
F_{\mathrm{Q}}(a)=F_{\mathrm{Q}}\left(\left[a^{-}, a^{+}\right]\right)=\lambda a^{+}+(1-\lambda) a^{-} .
$$

Definition 6 (see [47]). Based on the COWA operator in (7), we give a measure formula for IVIFSs as follows:

$$
E(\widetilde{A})=\frac{1}{n} \sum_{j=1}^{n} \frac{\min \left\{F_{\mathrm{Q}}\left(\mu_{\widetilde{a}_{j}}\right), F_{\mathrm{Q}}\left(v_{\widetilde{a}_{j}}\right)\right\}+\pi_{F_{\mathrm{Q}}\left(\widetilde{a}_{j}\right)}}{\max \left\{F_{\mathrm{Q}}\left(\mu_{\widetilde{a}_{j}}\right), F_{\mathrm{Q}}\left(v_{\widetilde{a}_{j}}\right)\right\}+\pi_{F_{\mathrm{Q}}\left(\widetilde{a}_{j}\right)}}
$$

where $\pi_{F_{Q}\left(\widetilde{a}_{j}\right)}=1-F_{Q}\left(\mu_{\widetilde{a}_{j}}\right)-F_{Q}\left(v_{\widetilde{a}_{j}}\right)$ and $\pi_{F_{Q}\left(\widetilde{a}_{j}\right)} \in[0,1], j=$ $1,2, \ldots, n$.

Definition 7 (see [39]). Let $\widetilde{a}\left(t_{1}\right), \widetilde{a}\left(t_{2}\right), \ldots, \widetilde{a}\left(t_{p}\right)$ be a collection of IVIFNs collected at $p$ different periods $t_{k}(k=$ $1,2, \ldots, p)$, and let $\lambda(t)=\left(\widetilde{a}\left(t_{1}\right), \widetilde{a}\left(t_{2}\right), \ldots, \widetilde{a}\left(t_{p}\right)\right)^{\mathrm{T}}$ be the weight vector of the periods $t_{k}(k=1,2, \ldots, p)$, then one calls

$$
\begin{aligned}
& \text { UDIVIFWA }_{\lambda(t)\left(\widetilde{a}\left(t_{1}\right), \tilde{a}\left(t_{2}\right), \ldots, \widetilde{a}\left(t_{p}\right)\right)} \\
& \quad=\lambda\left(t_{1}\right) \widetilde{a}\left(t_{1}\right) \oplus \lambda\left(t_{2}\right) \widetilde{a}\left(t_{2}\right) \oplus \cdots \oplus \lambda\left(t_{p}\right) \widetilde{a}\left(t_{p}\right)
\end{aligned}
$$

an uncertain dynamic interval-valued intuitionistic fuzzy weighted averaging (UDIVIFWA) operator, which can be rewritten as follows:

$$
\begin{aligned}
& \text { UDIVIFWA }_{\lambda(t)\left(\widetilde{a}\left(t_{1}\right), \tilde{a}\left(t_{2}\right), \ldots, \widetilde{a}\left(t_{p}\right)\right)} \\
& =\left(\left[\prod_{k=1}^{p}\left(\widetilde{\mu}_{\tilde{a}\left(t_{k}\right)}^{L}\right)^{\lambda\left(t_{k}\right)}, \prod_{k=1}^{p}\left(\widetilde{\mu}_{\widetilde{a}\left(t_{k}\right)}^{R}\right)^{\lambda\left(t_{k}\right)}\right],\right. \\
& \left.\left[1-\prod_{k=1}^{p}\left(1-\widetilde{v}_{\widetilde{a}\left(t_{k}\right)}^{L}\right)^{\lambda\left(t_{k}\right)}, 1-\prod_{k=1}^{p}\left(1-\widetilde{v}_{\tilde{a}\left(t_{k}\right)}^{R}\right)^{\lambda\left(t_{k}\right)}\right]\right) .
\end{aligned}
$$

\section{The Proposed Approach for Green Suppliers Selection}

4.1. Green Suppliers Problem Description. To the green supplier selection problem in construction projects, for which $S_{i}=\left\{S_{1}, S_{2}, \ldots, S_{m}\right\}(m \geq 2)$ is a discrete and feasible alternative solution set of green suppliers; $C_{j}=\left\{C_{1}, C_{2}, \ldots, C_{n}\right\}$ $(n \geq 2)$ is the finite set of criteria for green supplier selection in construction projects; $w=\left(w_{1}, w_{2}, \ldots, w_{n}\right)^{\mathrm{T}}$ is a weight vector, which satisfies $0 \leq w_{j} \leq 1, \sum_{j=1}^{n} w_{j}=1$; and $\lambda(t)=\left(\widetilde{a}\left(t_{1}\right), \widetilde{a}\left(t_{2}\right), \ldots, \widetilde{a}\left(t_{p}\right)\right)^{\mathrm{T}}$ is the time weight vector, where $0 \leq \lambda\left(t_{k}\right) \leq 1$ and $\sum_{k=1}^{p} \lambda\left(t_{k}\right)=1$. The value of criteria $C_{j}$, to which solution $S_{i}$ is subject at moment $t_{k}$, is denoted as $X_{i j}\left(t_{k}\right)$, which is subject to an interval-valued intuitionistic fuzzy, denoted as $X_{i j}\left(t_{k}\right) \sim \widetilde{a}_{i j}\left(t_{k}\right)=\left(\left[a_{i j}\left(t_{k}\right), b_{i j}\left(t_{k}\right)\right]\right.$, $\left.\left[c_{i j}\left(t_{k}\right), d_{i j}\left(t_{k}\right)\right]\right)$, forming an IVIF primitive green supplier selection matrix $D_{X_{i j}\left(t_{k}\right)}=\left(\left[a_{i j}\left(t_{k}\right), b_{i j}\left(t_{k}\right)\right],\left[c_{i j}\left(t_{k}\right)\right.\right.$, $\left.\left.d_{i j}\left(t_{k}\right)\right]\right)_{m \times n}$ based on $p$ moment of criteria for green supplier selection in construction projects. Green supplier selection problems consist of multiple dimensions, such as supplier, criteria, and time. Integration operator and determining time sequence weight are important technologies to reduce dimensionality and solve green supplier selection problem under interval-valued intuitionistic fuzzy environment.

\subsection{Interval-Valued Intuitionistic Fuzzy Geometric Heronian Means Operator}

Definition 8 (see [48]). Let $p \geq 0, q \geq 0$, and $p, q$ do not take the value 0 simultaneously. $a_{i}(i=1,2, \ldots, n)$ is a collection of nonnegative numbers. If

$$
\begin{aligned}
& \operatorname{GHM}^{p, q}\left(a_{1}, a_{2}, \ldots, a_{n}\right) \\
& =\frac{1}{p+q}\left(\prod_{i=1, j=1}^{n}\left(p a_{i}+q a_{j}\right)^{2 / n(n+1)}\right),
\end{aligned}
$$

then GHM is called the geometric Heronian means.

Definition 9. Let $\tilde{a}_{i}=\left(\left[a_{i}, b_{i}\right],\left[c_{i}, d_{i}\right]\right)(i=1,2, \ldots, n)$ be a group as IVIFNs, where $p \geq 0, q \geq 0$, then one calls

$$
\begin{aligned}
& \operatorname{IVIFGHM}^{p, q}\left(\tilde{a}_{1}, \tilde{a}_{2}, \ldots, \tilde{a}_{n}\right) \\
& \quad=\frac{1}{p+q}\left(\bigotimes_{i=1, j=1}^{n}\left(\left(p \tilde{a}_{i} \oplus q \tilde{a}_{j}\right)^{2 / n(n+1)}\right)\right)
\end{aligned}
$$

an interval-valued intuitionistic fuzzy geometric Heronian means (IVIFGHM) operator.

Theorem 10. Let $\tilde{a}_{i}=\left(\left[a_{i}, b_{i}\right],\left[c_{i}, d_{i}\right]\right)(i=1,2, \ldots, n)$ be a group of IVIFNs, where $p \geq 0, q \geq 0$, then the aggregated value by using the IVIFGHM is also an IVIFN, and

$$
\operatorname{IVIFGHM}^{p, q}\left(\tilde{a}_{1}, \tilde{a}_{2}, \ldots, \widetilde{a}_{n}\right)=([a, b],[c, d]),
$$

where

$$
\begin{aligned}
a & =1-(1 \\
& \left.-\prod_{i=1, j=1}^{n}\left(1-\left(1-a_{i}\right)^{p}\left(1-a_{j}\right)^{q}\right)^{2 / n(n+1)}\right)^{1 /(p+q)}, \\
b= & -(1 \\
& \left.-\prod_{i=1, j=1}^{n}\left(1-\left(1-b_{i}\right)^{p}\left(1-b_{j}\right)^{q}\right)^{2 / n(n+1)}\right)^{1 /(p+q)},
\end{aligned}
$$




$$
\begin{aligned}
& c=1-\left(1-\prod_{i=1, j=1}^{n}\left(1-\left(c_{i}\right)^{p}\left(c_{j}\right)^{q}\right)^{2 / n(n+1)}\right)^{1 /(p+q)}, \\
& d=1-\left(1-\prod_{i=1, j=1}^{n}\left(1-\left(d_{i}\right)^{p}\left(d_{j}\right)^{q}\right)^{2 / n(n+1)}\right)^{1 /(p+q)} .
\end{aligned}
$$

Proof. By the operational laws for IVIFNs, we have

$$
\begin{aligned}
& p a_{i}=\left(\left[1-\left(1-a_{i}\right)^{p}, 1-\left(1-b_{i}\right)^{p}\right],\left[c_{i}^{p}, d_{i}^{p}\right]\right), \\
& q a_{j}=\left(\left[1-\left(1-a_{j}\right)^{q}, 1-\left(1-b_{j}\right)^{q}\right],\left[c_{j}^{q}, d_{j}^{q}\right]\right), \\
& p \tilde{a}_{i} \oplus q \tilde{a}_{j}=\left(\left[1-\left(1-a_{i}\right)^{p}\left(1-a_{j}\right)^{q}, 1\right.\right. \\
&\left.\left.\quad-\left(1-b_{i}\right)^{p}\left(1-b_{j}\right)^{q}\right],\left[\left(c_{i}\right)^{p}\left(c_{j}\right)^{q},\left(d_{i}\right)^{p}\left(d_{j}\right)^{q}\right]\right) .
\end{aligned}
$$

Then

$$
\begin{aligned}
& \left(p \widetilde{a}_{i} \oplus q \widetilde{a}_{j}\right)^{2 / n(n+1)} \\
& =\left(\left[\left(1-\left(1-a_{i}\right)^{p}\left(1-a_{j}\right)^{q}\right)^{2 / n(n+1)},\right.\right. \\
& \left.\quad\left(1-\left(1-b_{i}\right)^{p}\left(1-b_{j}\right)^{q}\right)^{2 / n(n+1)}\right],[1 \\
& -\left(1-\left(c_{i}\right)^{p}\left(c_{j}\right)^{q}\right)^{2 / n(n+1)}, 1 \\
& \left.\left.\quad-\left(1-\left(d_{i}\right)^{p}\left(d_{j}\right)^{q}\right)^{2 / n(n+1)}\right]\right) .
\end{aligned}
$$

Let [49] $\beta_{i j}=\left(\left[t_{\beta_{i j}}, f_{\beta_{i j}}\right]\right)(i, j=1,2, \ldots, n)$ be an intuitionistic fuzzy number, then the operator can be defined as follows:

$$
\begin{aligned}
& \bigotimes_{i=1, j=1}^{n} \beta_{i j}^{2 / n(n+1)} \\
& \quad=\left(\prod_{i=1, j=1}^{n} t_{\beta_{i j} / n(n+1)}^{2} 1-\prod_{i=1, j=1}^{n}\left(1-f_{\beta_{i j}}\right)^{2 / n(n+1)}\right),
\end{aligned}
$$

where $t_{\beta_{i j}}$ defines the degree of membership of the element and $f_{\beta_{i j}}$ defines the degree of nonmembership.

Then replacing $\beta_{i j}, t_{\beta_{i j}}$, and $f_{\beta_{i j}}$ by $p \tilde{a}_{i} \oplus q \tilde{a}_{j}$, [ $(1-(1-$ $\left.\left.\left.a_{i}\right)^{p}\left(1-a_{j}\right)^{q}\right),\left(1-\left(1-b_{i}\right)^{p}\left(1-b_{j}\right)^{q}\right)\right]$, and $\left[\left(c_{i}\right)^{p}\left(c_{j}\right)^{q},\left(d_{i}\right)^{p}\left(d_{j}\right)^{q}\right]$ in (18), respectively, we have

$$
\begin{aligned}
& \bigotimes_{i=1, j=1}^{n}\left(p \tilde{a}_{i} \oplus q \tilde{a}_{j}\right)^{2 / n(n+1)} \\
& =\left(\left[\prod_{i=1, j=1}^{n}\left(1-\left(1-a_{i}\right)^{p}\left(1-a_{j}\right)^{q}\right)^{2 / n(n+1)},\right.\right.
\end{aligned}
$$

$$
\begin{aligned}
& \left.\prod_{i=1, j=1}^{n}\left(1-\left(1-b_{i}\right)^{p}\left(1-b_{j}\right)^{q}\right)^{2 / n(n+1)}\right],[1 \\
& -\prod_{i=1, j=1}^{n}\left(1-\left(c_{i}\right)^{p}\left(c_{j}\right)^{q}\right)^{2 / n(n+1)}, 1
\end{aligned}
$$$$
\left.\left.-\prod_{i=1, j=1}^{n}\left(1-\left(d_{i}\right)^{p}\left(d_{j}\right)^{q}\right)^{2 / n(n+1)}\right]\right) .
$$

Then

$$
\begin{aligned}
& \operatorname{IVIFGHM}^{p, q}\left(\widetilde{a}_{1}, \tilde{a}_{2}, \ldots, \widetilde{a}_{n}\right) \\
& \quad=\frac{1}{p+q}\left(\bigotimes_{i=1, j=1}^{n}\left(\left(p \widetilde{a}_{i} \oplus q \tilde{a}_{j}\right)^{2 / n(n+1)}\right)\right) \\
& \quad=([a, b],[c, d]),
\end{aligned}
$$

where

$$
\begin{aligned}
a & =1-(1 \\
& \left.-\prod_{i=1, j=1}^{n}\left(1-\left(1-a_{i}\right)^{p}\left(1-a_{j}\right)^{q}\right)^{2 / n(n+1)}\right)^{1 /(p+q)}, \\
b= & -\left(1-\prod_{i=1, j=1}^{n}\left(1-\left(1-b_{i}\right)^{p}\left(1-b_{j}\right)^{q}\right)^{2 / n(n+1)}\right)^{1 /(p+q)}, \\
& -1-\left(1-\prod_{i=1, j=1}^{n}\left(1-\left(c_{i}\right)^{p}\left(c_{j}\right)^{q}\right)^{2 / n(n+1)}\right)^{1 /(p+q)}, \\
d & =1-\left(1-\prod_{i=1, j=1}^{n}\left(1-\left(d_{i}\right)^{p}\left(d_{j}\right)^{q}\right)^{2 / n(n+1)}\right)^{1 /(p+q)},
\end{aligned}
$$

which completes the proof of Theorem 10 .

The IVIFGHM considers the interaction between criteria for green supplier selection in construction projects, but it has different levels of importance in green supplier selection. Therefore, we first propose an interval-valued intuitionistic fuzzy geometric weighted Heronian means (IVIFGWHM) operator. 
Definition 11. Let $\tilde{a}_{i}=\left(\left[a_{i}, b_{i}\right],\left[c_{i}, d_{i}\right]\right)(i=1,2, \ldots, n)$ be a group of IVIFNs, where $p \geq 0, q \geq 0$, and $w=\left(w_{1}\right.$, $\left.w_{2}, \ldots, w_{n}\right)^{\mathrm{T}}$ is the weight vector, which satisfies $0 \leq w_{j} \leq$ $1, \sum_{j=1}^{n} w_{j}=1$, and then one calls

$$
\begin{aligned}
& \operatorname{IVIFGWHM}^{p, q}\left(\tilde{a}_{1}, \tilde{a}_{2}, \ldots, \tilde{a}_{n}\right) \\
& \quad=\frac{1}{p+q}\left(\bigotimes_{i=1, j=1}^{n}\left(\left(\left(p \widetilde{a}_{i}\right)^{w_{i}} \oplus\left(q \widetilde{a}_{j}\right)^{w_{j}}\right)^{2 / n(n+1)}\right)\right)
\end{aligned}
$$

an interval-valued intuitionistic fuzzy geometric weighted Heronian means (IVIFGWHM) operator.

Theorem 12. Let $\tilde{a}_{i}=\left(\left[a_{i}, b_{i}\right],\left[c_{i}, d_{i}\right]\right)(i=1,2, \ldots, n)$ be $a$ group of IVIFNs, where $p \geq 0, q \geq 0$, and $w=\left(w_{1}, w_{2}, \ldots\right.$, $\left.w_{n}\right)^{T}$ is the weight vector, which satisfies $0 \leq w_{j} \leq 1$, $\sum_{j=1}^{n} w_{j}=1$, then the aggregated value by using the IVIFGWHM is also an IVIFN, and

$$
\operatorname{IVIFGWHM}^{p, q}\left(\tilde{a}_{1}, \tilde{a}_{2}, \ldots, \tilde{a}_{n}\right)=\left(\left[a^{\prime}, b^{\prime}\right],\left[c^{\prime}, d^{\prime}\right]\right),
$$

where

$$
\begin{aligned}
a^{\prime}= & 1-\left(1-\prod_{i=1, j=1}^{n}\left(1-\left(1-\left(a_{i}\right)^{w_{i}}\right)^{p}\right.\right. \\
& \left.\left.\cdot\left(1-\left(a_{j}\right)^{w_{j}}\right)^{q}\right)^{2 / n(n+1)}\right)^{1 /(p+q)}, \\
b^{\prime}= & 1-\left(1-\prod_{i=1, j=1}^{n}\left(1-\left(1-\left(b_{i}\right)^{w_{i}}\right)^{p}\right.\right. \\
& \left.\left.\cdot\left(1-\left(b_{j}\right)^{w_{j}}\right)^{q}\right)^{2 / n(n+1)}\right)^{1 /(p+q)}, \\
c^{\prime}= & \left(1-\prod_{i=1, j=1}^{n}\left(1-\left(1-\left(1-c_{i}\right)^{w_{i}}\right)^{p}\right.\right. \\
& \left.\left.\cdot\left(1-\left(1-c_{j}\right)^{w_{j}}\right)^{q}\right)^{2 / n(n+1)}\right)^{1 /(p+q)}, \\
d^{\prime}= & \left(1-\prod_{i=1, j=1}^{n}\left(1-\left(1-\left(1-d_{i}\right)^{w_{i}}\right)^{p}\right.\right. \\
& \left.\left.\left.\left(1-d_{j}\right)^{w_{j}}\right)^{q}\right)^{2 / n(n+1)}\right)^{1 /(p+q)}
\end{aligned}
$$

Proof. The proof of Theorem 12 is similar to Theorem 10; we do not duplicate here.
4.3. Determination of Comprehensive Time Sequence Weight Based on Ideal Solution and Information Entropy. During the process of green supplier selection in construction projects, time sequence weight vector, which reflects constructor's preference degree towards time series, $\lambda(t)=\left(\widetilde{a}\left(t_{1}\right), \widetilde{a}\left(t_{2}\right)\right.$, $\left.\ldots, \widetilde{a}\left(t_{p}\right)\right)^{\mathrm{T}}$, is vital to assemble time sequence information and obtain an accurate selection result.

\subsubsection{Time Weight Based on Time Degree and Ideal Solution}

Definition 13 (see [41]). Let $\varphi=\sum_{k=1}^{p}((p-k) /(p-1)) \lambda\left(t_{k}\right)$ be a time degree vector $\lambda(t)=\left(\widetilde{a}\left(t_{1}\right), \widetilde{a}\left(t_{2}\right), \ldots, \widetilde{a}\left(t_{p}\right)\right)^{\mathrm{T}}$, where $0 \leq \varphi \leq 1$.

Time degree indicates the concern and preference degree of decision-maker towards time series. Based on this analysis, when $\varphi=0$, then $\lambda(t)^{+}=(0,0, \ldots, 1)^{\mathrm{T}}$, which is called positive ideal time weight vector, and the decision-maker only attaches current information; when $\varphi$ increases, the decision-maker attaches more importance to older information; when $\varphi=1$, then $\lambda(t)^{-}=(1,0, \ldots, 0)^{\mathrm{T}}$, which is called negative ideal time weight vector, and the decision-maker only attaches older information.

Let $d\left(\lambda^{1}\left(t_{k}\right), \lambda^{2}\left(t_{k}\right)\right)$ be an Euclidean distance between time weight vectors $\lambda^{1}\left(t_{k}\right)$ and $\lambda^{2}\left(t_{k}\right)$; the distance can be denoted as follows:

$$
d\left(\lambda^{1}\left(t_{k}\right), \lambda^{2}\left(t_{k}\right)\right)=\sqrt{\sum_{k=1}^{p}\left|\lambda^{1}\left(t_{k}\right)-\lambda^{2}\left(t_{k}\right)\right|^{2}}
$$

Thus, the Euclidean distance of the time weight vector $\lambda(t)=\left(\widetilde{a}\left(t_{1}\right), \widetilde{a}\left(t_{2}\right), \ldots, \widetilde{a}\left(t_{p}\right)\right)^{\mathrm{T}}$ from the positive and negative ideal time weight vector can be denoted as follows:

$$
\begin{aligned}
& d\left(\lambda\left(t_{k}\right), \lambda\left(t_{k}\right)^{+}\right)=\sqrt{\sum_{k=1}^{p-1} \lambda\left(t_{k}\right)^{2}+\left(1-\lambda\left(t_{p}\right)\right)^{2}} \\
& d\left(\lambda\left(t_{k}\right), \lambda\left(t_{k}\right)^{-}\right)=\sqrt{\left(1-\lambda\left(t_{1}\right)\right)^{2}+\sum_{k=2}^{p} \lambda\left(t_{k}\right)^{2}} .
\end{aligned}
$$

The ideal time weight vector can be denoted as follows:

$$
C=\frac{d\left(\lambda\left(t_{k}\right), \lambda\left(t_{k}\right)^{-}\right)}{d\left(\lambda\left(t_{k}\right), \lambda\left(t_{k}\right)^{+}\right)+d\left(\lambda\left(t_{k}\right), \lambda\left(t_{k}\right)^{-}\right)} .
$$

Then, a model can be optimised based on TOPSIS thought and ideal solution. The model (M-1) can be denoted as follows:

$$
\max C(\lambda)=\frac{\sqrt{\left(1-\lambda\left(t_{1}\right)\right)^{2}+\sum_{k=2}^{p} \lambda\left(t_{k}\right)^{2}}}{\sqrt{\sum_{k=1}^{p-1} \lambda\left(t_{k}\right)^{2}+\left(1-\lambda\left(t_{p}\right)\right)^{2}}+\sqrt{\left(1-\lambda\left(t_{1}\right)\right)^{2}+\sum_{k=2}^{p} \lambda\left(t_{k}\right)^{2}}}
$$




$$
\text { s.t. } \varphi=\sum_{k=1}^{p} \frac{p-k}{p-1} \lambda\left(t_{k}\right), \quad \sum_{k=1}^{p} \lambda\left(t_{k}\right)=1, \lambda\left(t_{k}\right) \in[0,1], k=1,2, \ldots, p \text {. }
$$

4.3.2. Time Weight Based on Time Degree and Information Entropy. Based on the theory of information entropy, the greater the entropy is, the less information quantity it contains. Information entropy reflects the uptake degree of time weight vector against information quantity, which has some characteristics, such as symmetry, additivity, and extremum. The expression is as follows:

$$
F\left(\lambda\left(t_{k}\right)\right)=-\sum_{k=1}^{p} \lambda\left(t_{k}\right) \ln \lambda\left(t_{k}\right), \quad k=1,2, \ldots, p
$$

Then, based on maximum entropy principle, solve the time weight of information entropy and set up a nonlinear programming model (M-2) as follows:

$$
\max F\left(\lambda\left(t_{k}\right)\right)=-\sum_{k=1}^{p} \lambda\left(t_{k}\right) \ln \lambda\left(t_{k}\right)
$$

$$
\begin{aligned}
& \text { s.t. } \quad \varphi=\sum_{k=1}^{p} \frac{p-k}{p-1} \lambda\left(t_{k}\right), \\
& \quad \sum_{k=1}^{p} \lambda\left(t_{k}\right)=1, \lambda\left(t_{k}\right) \in[0,1], k=1,2, \ldots, p .
\end{aligned}
$$

4.3.3. Comprehensive Time Weight. By the principle of "stress the present rather than the past," the greater the closeness degree is, the more the attention is attached to current decision-making information. To maximise the closeness of a time weight vector, a model is optimised based on (M-1) and (M-2) models; this paper constructs a nonlinear programming model (M-3), which combines information entropy principle and ideal solution. It considers the influence of constructors subjective preference and objective criteria information and can make the green supplier selection result in construction projects more comprehensive, accurate, and reliable. The model (M-3) can be denoted as follows:

$$
\begin{aligned}
\max \quad G & =\theta \frac{\sqrt{\left(1-\lambda\left(t_{1}\right)\right)^{2}+\sum_{k=2}^{p} \lambda\left(t_{k}\right)^{2}}}{\sqrt{\sum_{k=1}^{p-1} \lambda\left(t_{k}\right)^{2}+\left(1-\lambda\left(t_{p}\right)\right)^{2}}+\sqrt{\left(1-\lambda\left(t_{1}\right)\right)^{2}+\sum_{k=2}^{p} \lambda\left(t_{k}\right)^{2}}}-(1-\theta) \sum_{k=1}^{p} \lambda\left(t_{k}\right) \ln \lambda\left(t_{k}\right) \\
\text { s.t. } \quad \varphi & =\sum_{k=1}^{p} \frac{p-k}{p-1} \lambda\left(t_{k}\right), \quad \sum_{k=1}^{p} \lambda\left(t_{k}\right)=1, \lambda\left(t_{k}\right) \in[0,1], k=1,2, \ldots, p,
\end{aligned}
$$

where $\theta$ is a balance coefficient between the above (M-1) and (M-2) models, $\theta \in[0,1]$, the different values of parameter $\theta$ specify that constructors lay particular stress on one of them. The model (M-3) can be solved via Lingo software, from which time sequence weight based on ideal solution vector $\lambda(t)=\left(\widetilde{a}\left(t_{1}\right), \tilde{a}\left(t_{2}\right), \ldots, \tilde{a}\left(t_{p}\right)\right)^{\mathrm{T}}$ is acquired.

4.4. Steps of Green Supplier Selection in Construction Projects. According to the calculation process of the above model for green supplier selection in construction projects, the calculation steps based on IVIFGWHM and time sequence are as follows.

Step 1. The original information matrix $D_{X_{i j}\left(t_{k}\right)}=\left(\left[a_{i j}\left(t_{k}\right)\right.\right.$, $\left.\left.b_{i j}\left(t_{k}\right)\right],\left[c_{i j}\left(t_{k}\right), d_{i j}\left(t_{k}\right)\right]\right)_{m \times n}$ of green supplier selection is given by construction project managers, practitioners, and industry experts, based on $p$ moments according to the plan set, criteria set, and time sequence set.

Step 2. Based on the main criteria of green supplier selection for constructor in Table 1, we form the criteria weight set $w=\left(w_{1}, w_{2}, \ldots, w_{n}\right)^{\mathrm{T}}$ according to Formula (9) and calculate the time sequence weight set $\lambda(t)=\left(\widetilde{a}\left(t_{1}\right), \tilde{a}\left(t_{2}\right), \ldots, \widetilde{a}\left(t_{p}\right)\right)^{\mathrm{T}}$ according to Formula (33).

Step 3. Utilize the IVIFGWHM operator to aggregate the criteria information of green supplier selection based on the criteria weight which is calculated in Step 2. Then, we need to aggregate all individual criteria information $C_{j}$ potential green suppliers into a collective criteria information matrix $D_{X_{i j}\left(t_{k}\right)}^{\prime}=\left(\left[a_{i j}^{\prime}\left(t_{k}\right), b_{i j}^{\prime}\left(t_{k}\right)\right],\left[c_{i j}^{\prime}\left(t_{k}\right), d_{i j}^{\prime}\left(t_{k}\right)\right]\right)_{m \times n}$ according to Formula (25).

Step 4. Apply Formula (11) to gather the information of time dimension of green supplier selection based on the time sequence weight set $\lambda(t)=\left(\widetilde{a}\left(t_{1}\right), \widetilde{a}\left(t_{2}\right), \ldots, \widetilde{a}\left(t_{p}\right)\right)^{\mathrm{T}}$. Then, we create the comprehensive decision information matrix $D_{X_{i}}^{\prime \prime}=\left(\left[a_{i}^{\prime \prime}, b_{i}^{\prime \prime}\right],\left[c_{i}^{\prime \prime}, d_{i}^{\prime \prime}\right]\right)_{m \times 1}$ for the single dimension to potential green suppliers $S_{i}$.

Step 5. Finally, select the best green supplier in construction projects based on ranking value $L_{i}=\left(L_{1}, L_{2}, \ldots, L_{i}\right)$ and 
further determine the priority sequence of green supplier $S_{i}(i=1,2, \ldots, m)$.

\section{Case Study}

5.1. Case Company Background. According to the above analysis, the proposed method is applied on the case of the housing construction project entity in the construction industry to solve green supplier selection problem.

Company $H$, founded in 1952 , is a builder enterprise, which has special qualifications for construction, located in Harbin, a city of Heilongjiang Province in China. H's business scope involves housing construction general contracting, infrastructure construction, real estate investment, engineering design, and other fields in the major cities in all provinces of China, Africa, South Asia, and Southeast Asia. H will be committed to green housing technology development and practice, with product innovation and the provision of green building products as the development goal.

At present, the concept of green building in China is gradually taken seriously and the government has set the target of carbon emissions reduction for the construction enterprises in China. If the total carbon emissions of constructors exceed the carbon emissions limit set by the government, then they will face huge fines from government agencies. For builder $H$, one of the important issues is how to reduce carbon emissions of construction projects to enhance green competitiveness and profit. In these circumstances, $H$ needs to select its green supplier from a large number of suppliers in construction projects.

As builder $H$ has some experience accumulation in the supplier selection, it is still a difficult problem for $H$ to select its best green supplier from these potential suppliers in construction projects. On the one hand, builder $H$ has established a criterion, which is not appropriate to use to select green supplier. It did not establish the criteria for the green supplier selection in construction projects. On the other hand, builder $H$ not only has to nondimensionalize the criteria to previous supplier selection, but also focus more on the single period of decision criteria information, and even if $H$ considers multiple timings during supplier selection, it may still lead to a subjectivity and objectivity in the time weight. Moreover, the selection method is very difficult for builder $H$ to deal with qualitative criteria in the process of green supplier selection.

5.2. Application of the Proposed Criteria and Method. To builder $H$, the proposed criteria and method are suitable to be used to select green supplier in construction projects, because the managers and practitioners' understanding of the weights of criteria for green supplier selection is in the fuzzy state in builder $H$. In addition, expert scoring method, which is usually used to select traditional supplier in their construction projects, makes the proposed criteria and method more realistic and practical.

For the moment, builder $H$ is required to purchase a batch of rebar for a green building in Harbin. After the primary selection of steel production enterprises, there are four enterprises $S_{i}=\left\{S_{1}, S_{2}, S_{3}, S_{4}\right\}$ to enter the final selection. Builder $H$ needs to select its steel supplier from 4 main green suppliers by the proposed criteria and method. Therefore, H's 15 managers, practitioners, and experts are asked to determine the criteria of green supplier selection to construction projects based on the preliminary list of criteria compiled including literature review about green supplier selection and the builder $H$ 's actual situation. It can be seen in Table 1 including 4 main criteria (building materials information, green business operation, potential for sustainable cooperation, and green technology capability) and 17 subcriteria (e.g., building materials price and green and waste materials reclamation). Moreover, they select the time sequence set of different historical periods nearly three years, $t_{k}=\left(t_{1}, t_{2}, t_{3}\right)$, for the previously mentioned potential green suppliers. For the sake of simplicity, we only give out the calculation for the 4 main criteria. The evaluated values of 4 main suppliers, which are given by 15 managers, practitioners, and experts, are listed in Tables 2-4.

Based on the original evaluation criteria information matrix of green supplier selection which only includes supplier $S_{1}$, supplier $S_{2}$, supplier $S_{3}$, and supplier $S_{4}$ in construction projects, per Step 2, according to Formula (9), the criteria weight is shown in Table 5. According to Formula (33), the time degree parameter $\varphi=0.3$ and the discrete time weight vector, which is solved via Step 2 and Lingoll.0 software, are shown in Table 5.

Based on the criteria weight vector $w=\left(w_{1}, w_{2}, w_{3}, w_{4}\right)$, per Step 3, according to Formula (25), where $p=q=$ 1 , the four-criterion information at different moments was integrated into a comprehensive criteria information matrix, respectively, as shown in Table 6 .

Comprehensive criteria making information of each potential green supplier was assembled from different moment, per Step 4, forming comprehensive selection information matrix for the target single dimension to potential green suppliers in construction projects calculated as

$$
\begin{aligned}
& S_{1}=([0.6893,0.7679],[0.1089,0.1626]), \\
& S_{2}=([0.7470,0.8276],[0.0654,0.1131]), \\
& S_{3}=([0.7598,0.5632],[0.0713,0.1261]), \\
& S_{4}=([0.7217,0.8190],[0.0849,0.1280]) .
\end{aligned}
$$

In the end, the value of each potential green supplier for the construction project was determined based on Step 5:

$$
\begin{aligned}
L_{i} & =\left(L_{1}, L_{2}, L_{3}, L_{4}\right) \\
& =(0.6928,0.7693,0.6254,0.7469) .
\end{aligned}
$$

The priority sequence obtained from the ranking value of the four potential green suppliers is $S_{2}>S_{4}>S_{1}>S_{3}$. Thus, the green supplier, which will provide the batch of rebar for the green building in Harbin, is determined to be $S_{2}$.

Further, for potential green supplier selection, the dynamic multicriteria decision-making solution operator of criteria information and time weight were also calculated using the possibility different method described in the literature $[38,44]$. 
TABLE 2: Original evaluation criteria information matrix at the moment $t_{1}$.

\begin{tabular}{lcccc}
\hline & $C_{1}$ & $C_{2}$ & $C_{3}$ & $C_{4}$ \\
\hline$S_{1}$ & $([0.3,0.4],[0.3,0.5])$ & $([0.1,0.2],[0.5,0.6])$ & $([0.1,0.2],[0.7,0.7])$ & $([0.2,0.3],[0.3,0.5])$ \\
$S_{2}$ & $([0.6,0.7],[0.2,0.3])$ & $([0.4,0.5],[0.1,0.2])$ & $([0.4,0.6],[0.3,0.4])$ & $([0.1,0.2],[0.2,0.5])$ \\
$S_{3}$ & $([0.5,0.5],[0.2,0.5])$ & $([0.4,0.5],[0.2,0.3])$ & $([0.2,0.3],[0.5,0.6])$ & $([0.4,0.5],[0.1,0.2])$ \\
$S_{4}$ & $([0.1,0.2],[0.6,0.7])$ & $([0.3,0.4],[0.5,0.5])$ & $([0.2,0.4],[0.1,0.2])$ & $([0.6,0.8],[0.1,0.2])$ \\
\hline
\end{tabular}

TABLE 3: Original evaluation criteria information matrix at the moment $t_{2}$.

\begin{tabular}{llccc}
\hline & $C_{1}$ & $C_{2}$ & $C_{3}$ & $C_{4}$ \\
\hline$S_{1}$ & $([0.3,0.4],[0.5,0.6])$ & $([0.4,0.5],[0.2,0.4])$ & $([0.4,0.5],[0.3,0.5])$ & $([0.3,0.5],[0.2,0.4])$ \\
$S_{2}$ & $([0.1,0.2],[0.6,0.7])$ & $([0.5,0.6],[0.2,0.3])$ & $([0.4,0.7],[0.1,0.2])$ & $([0.4,0.7],[0.1,0.3])$ \\
$S_{3}$ & $([0.2,0.6],[0.3,0.4])$ & $([0.4,0.4],[0.2,0.4])$ & $([0.3,0.4],[0.3,0.6])$ & $([0.5,0.6],[0.1,0.3])$ \\
$S_{4}$ & $([0.4,0.5],[0.3,0.4])$ & $([0.5,0.5],[0.1,0.4])$ & $([0.5,0.6],[0.2,0.4])$ & $([0.3,0.5],[0.3,0.4])$ \\
\hline
\end{tabular}

TABLE 4: Original evaluation criteria information matrix at the moment $t_{3}$.

\begin{tabular}{llccc}
\hline & $C_{1}$ & $C_{2}$ & $C_{3}$ & $C_{4}$ \\
\hline$S_{1}$ & $([0.3,0.5],[0.2,0.4])$ & $([0.7,0.8],[0.1,0.2])$ & $([0.3,0.4],[0.2,0.3])$ & $([0.4,0.6],[0.2,0.4])$ \\
$S_{2}$ & $([0.3,0.6],[0.1,0.3])$ & $([0.4,0.5],[0.3,0.4])$ & $([0.2,0.3],[0.4,0.5])$ & $([0.4,0.7],[0.2,0.3])$ \\
$S_{3}$ & $([0.2,0.7],[0.2,0.2])$ & $([0.2,0.4],[0.2,0.4])$ & $([0.5,0.7],[0.2,0.3])$ & $([0.3,0.4],[0.3,0.5])$ \\
$S_{4}$ & $([0.3,0.5],[0.1,0.4])$ & $([0.3,0.4],[0.2,0.4])$ & $([0.1,0.6],[0.3,0.4])$ & $([0.2,0.7],[0.2,0.3])$ \\
\hline
\end{tabular}

TABle 5: Criteria weight and discrete time weight.

\begin{tabular}{cccccc}
\hline & $\lambda(t)$ & $w\left(t_{1}\right)$ & $w\left(t_{2}\right)$ & $w\left(t_{3}\right)$ & $w\left(t_{4}\right)$ \\
\hline$t_{1}$ & 0.582 & 0.2534 & 0.2342 & 0.2579 & 0.2545 \\
$t_{2}$ & 0.236 & 0.2574 & 0.2492 & 0.2433 & 0.2501 \\
$t_{3}$ & 0.182 & 0.2550 & 0.2454 & 0.2284 & 0.2712 \\
\hline
\end{tabular}

First, in [38], $\mathrm{Xu}$ and Chen proposed a method of interval-valued intuitionistic fuzzy number weighted arithmetic average (IVIFNWAA) operator. To compare Xu and Chen's approach with the proposed method, here we utilize it to aggregate criteria information of each potential green supplier at different moment. The four criteria were assembled into a collective selection criteria information at different moment shown in Table 7.

The following ranking value of each potential green suppliers for the construction project was acquired based on the IVIFNWAA operator and time sequence weight which is proposed in the paper. According to Steps 2-5, the final rank values are

$$
\begin{aligned}
L_{i}^{\prime} & =\left(L_{1}, L_{2}, L_{3}, L_{4}\right) \\
& =(0.0320,0.3266,0.2618,0.2699) .
\end{aligned}
$$

The priorities are $S_{2}>S_{4}>S_{3}>S_{1}$, where $S_{2}$ is the optimal green supplier for this construction project.
Then, in [44], Xu proposed a BUM function method. To compare Xu's approach with the proposed method, we further determine the time sequence weight as follows:

$$
\begin{aligned}
\lambda(t) & =\left(\lambda\left(t_{1}\right), \lambda\left(t_{2}\right), \lambda\left(t_{3}\right)\right) \\
& =(0.1111,0.3333,5556) .
\end{aligned}
$$

And, the following ranking value was acquired based on the IVIFGWHM operator and BUM function method. The final rank values are

$$
\begin{aligned}
L_{i}^{\prime \prime} & =\left(L_{1}, L_{2}, L_{3}, L_{4}\right) \\
& =(0.7824,0.7737,0.5775,0.7661) .
\end{aligned}
$$

The priorities are $S_{1}>S_{2}>S_{4}>S_{3}$, where $S_{1}$ is the optimal green supplier for this construction project.

Obviously, the result based on the method described in the literature $[38,44]$ proves the rationality of the method proposed for green suppliers selection in construction projects in this study. Moreover, compared with similar dynamic multicriteria decision-making methods for green suppliers selection, the proposed method considers the interaction between criteria under time sequence. Thus, the final ordering result is more comprehensive and reasonable. The method proposed in this paper can help constructors to identify the target and reduce the time cost of green suppliers selection in construction projects.

Based on the evaluation and selection above, supplier $S_{2}$ is recommended as builder $H$ 's best green supplier. In fact, 
TABLE 6: Comprehensive selection criteria information based on IVIFGWHM operator.

\begin{tabular}{llcc}
\hline & $t_{1}$ & $t_{2}$ & $t_{3}$ \\
\hline$S_{1}$ & $([0.6314,0.7174],[0.1517,0.1969])$ & $([0.7668,0.8289],[0.0911,0.1522])$ & $([0.7948,0.8645],[0.0475,0.0960])$ \\
$S_{2}$ & $([0.7489,0.8195],[0.0565,0.1073])$ & $([0.7395,0.8345],[0.0865,0.1289])$ & $([0.7507,0.8449],[0.0724,0.1127])$ \\
$S_{3}$ & $([0.7717,0.8136],[0.0779,0.1301])$ & $([0.7583,0.1655],[0.0637,0.1335])$ & $([0.7250,0.8502],[0.0623,0.1059])$ \\
$S_{4}$ & $([0.7050,0.7953],[0.1089,0.1372])$ & $([0.8028,0.8502],[0.0640,0.1199])$ & $([0.6771,0.8571],[0.0553,0.1115])$ \\
\hline
\end{tabular}

TABLE 7: Comprehensive selection criteria information based on IVIFNWAA operator.

\begin{tabular}{llcr}
\hline & $t_{1}$ & $t_{2}$ & $t_{3}$ \\
\hline$S_{1}$ & $([0.1805,0.2811],[0.4207,0.5691])$ & $([0.3512,0.4760],[0.2795,0.4688])$ & $([0.4547,0.6081],[0.1687,0.3160])$ \\
$S_{2}$ & $([0.3997,0.5326],[0.1888,0.3346])$ & $([0.3636,0.5851],[0.1885,0.3381])$ & $([0.3336,0.5559],[0.2169,0.3618])$ \\
$S_{3}$ & $([0.3830,0.4547],[0.2123,0.3683])$ & $([0.3591,0.5116],[0.2060,0.4108])$ & $([0.3070,0.5708],[0.2232,0.3335])$ \\
$S_{4}$ & $([0.3303,0.5120],[0.2296,0.3405])$ & $([0.4300,0.5264],[0.2067,0.4000])$ & $([0.2313,0.5674],[0.1839,0.3700])$ \\
\hline
\end{tabular}

builder $H$ has given priority to supplier $S_{2}$, which provides the batch of rebar for the green building in Harbin, according to the results. In addition, supplier $S_{4}$ is recommended as the reserved green supplier. $H$ 's green housing technology development and practice will improve its competitiveness and profitability in the construction industry based on the concept of continuous improvement.

\section{Conclusions}

There has been broad consensus on carbon emissions reduction around the world. Green building can not only bring a healthier and more comfortable living environment, but also reduce carbon emissions in the construction industry. For constructors, use of green building materials for construction and sustainable development of the environment is particularly important. In addition, GSCM has become an inevitable choice for constructors to cope with the pressure from the government and the market. Therefore, it is one of the most important factors to select green supplier in construction projects.

In this paper, we propose a dynamic multiattribute decision-making approach with interval-valued intuitionistic fuzzy for green supplier selection in construction projects. According to the demand of constructors in construction projects, 4 main criteria and 17 subcriteria are established for green supplier selection in the construction industry. The proposed method considers interaction between criteria of green supplier selection and the influence of constructors subjective preference and objective criteria information. The evaluated values of potential green suppliers are given by managers, practitioners, and experts. The proposed criteria and method are suitable to use to select green supplier in construction projects because the managers and practitioners' understanding of the weights of criteria for green supplier selection is in the intuitionistic fuzzy due to the nature of unquantifiable and incomplete information in green supplier selection. In addition, expert scoring method, which is usually used to select traditional supplier in their construction projects, make the proposed criteria and method more realistic and practical. The proposed criteria and method have been successfully implemented in a case construction project to select the best green supplier. It not only is much more easier for constructors to select green supplier, but also can make the localization of green supplier more practical and more accurate in the construction industry. Finally, green supplier selection of builder $H$ for a green building in Harbin is studied to verify the scientificity and feasibility of the proposed criteria and method. The result shows that these criteria and method are of effectiveness and practicality of green supplier selection in construction projects. Also, it can be mentioned that the proposed model can be easily extended to analyze other management decision problems as a structural model.

For further study, we will extend the proposed method in this paper with interval-valued intuitionistic trapezoidal fuzzy numbers and prospect theory in other engineering fields.

\section{Conflicts of Interest}

The authors declare that they have no conflicts of interest.

\section{Acknowledgments}

This research was supported by the National Fund Key Project on Social Science of China (14AGL004), the National Natural Science Foundation of China (71073034), and the Fundamental Research Funds for the Central Universities (HEUCFW170901).

\section{References}

[1] J. Yudelson, Green Building A to Z: Understanding the Language of Green Building, New Society Publishers, Gabriola Island, BC, Canada, 2007.

[2] Change I P O C, Climate change 2014 synthesis report, Environmental Policy Collection, 2014.

[3] B. Kang, Y. Hu, Y. Deng, and D. Zhou, "A new methodology of multicriteria decision-making in supplier selection based on z-numbers," Mathematical Problems in Engineering, vol. 2016, Article ID 8475987, 17 pages, 2016.

[4] A. H. I. Lee, H. Kang, C. F. Hsu, and H. Hung, "A green supplier selection model for high-tech industry," Expert Systems with Applications, vol. 36, no. 4, pp. 7917-7927, 2009. 
[5] C.-W. Hsu, T.-C. Kuo, S.-H. Chen, and A. H. Hu, "Using DEMATEL to develop a carbon management model of supplier selection in green supply chain management," Journal of Cleaner Production, vol. 56, pp. 164-172, 2013.

[6] D. Kannan and C. J. C. Jabbour, "Selecting green suppliers based on GSCM practices: using fuzzy TOPSIS applied to a Brazilian electronics company," European Journal of Operational Research, vol. 233, no. 2, pp. 432-447, 2014.

[7] C.-W. Tsui and U.-P. Wen, "A hybrid multiple criteria group decision-making approach for green supplier selection in the TFT-LCD industry," Mathematical Problems in Engineering, vol. 2014, Article ID 709872, 13 pages, 2014.

[8] O. Gurel, A. Z. Acar, I. Onden, and I. Gumus, "Determinants of the green supplier selection," Procedia: Social and Behavioral Sciences, vol. 181, pp. 131-139, 2015.

[9] H. M. W. Chen, S.-Y. Chou, Q. D. Luu, and T. H.-K. Yu, "A fuzzy MCDM approach for green supplier selection from the economic and environmental aspects," Mathematical Problems in Engineering, vol. 2016, Article ID 8097386, 10 pages, 2016.

[10] F. Yu, Y. Yang, and D. Chang, "Carbon footprint based green supplier selection under dynamic environment," Journal of Cleaner Production, vol. 170, pp. 880-889, 2018.

[11] K. Govindan and R. Sivakumar, "Green supplier selection and order allocation in a low-carbon paper industry: integrated multi-criteria heterogeneous decision-making and multi-objective linear programming approaches," Annals of Operations Research, vol. 238, no. 1-2, pp. 243-276, 2016.

[12] Q. Pang, T. Yang, M. Li, and Y. Shen, "A fuzzy-grey multicriteria decision making approach for green supplier selection in lowcarbon supply chain," Mathematical Problems in Engineering, vol. 2017, Article ID 9653261, 9 pages, 2017.

[13] X. Zhang, L. Shen, and Y. Wu, "Green strategy for gaining competitive advantage in housing development: a China study," Journal of Cleaner Production, vol. 19, no. 2-3, pp. 157-167, 2011.

[14] S. Mokhlesian, "How do contractors select suppliers for greener construction projects? The case of three Swedish companies," Sustainability, vol. 6, no. 7, pp. 4133-4151, 2014.

[15] I. Ruuska, T. Ahola, M. Martinsuo, and T. Westerholm, "Supplier capabilities in large shipbuilding projects," International Journal of Project Management, vol. 31, no. 4, pp. 542-553, 2013.

[16] C. Woo, M. G. Kim, Y. Chung, and J. J. Rho, “Suppliers' communication capability and external green integration for green and financial performance in Korean construction industry," Journal of Cleaner Production, vol. 112, pp. 483-493, 2016.

[17] M. G. Kim, C. Woo, J. J. Rho, and Y. Chung, "Environmental capabilities of suppliers for green supply chain management in construction projects: A Case Study in Korea," Sustainability, vol. 8, no. 1, pp. 1-17, 2016.

[18] A. P. Chan, A. Darko, and E. E. Ameyaw, "Strategies for promoting green building technologies adoption in the construction industry-An international study," Sustainability, vol. 9, no. 6, p. 969, 2017.

[19] K. Govindan, R. Khodaverdi, and A. Jafarian, "A fuzzy multi criteria approach for measuring sustainability performance of a supplier based on triple bottom line approach," Journal of Cleaner Production, vol. 47, pp. 345-354, 2013.

[20] B. P. Saradhi, N. R. Shankar, and C. Suryanarayana, "Novel Distance Measure in Fuzzy TOPSIS for Supply Chain Strategy Based Supplier Selection," Mathematical Problems in Engineering, vol. 2016, Article ID 7183407, 17 pages, 2016.
[21] S. Darabi and J. Heydari, "An interval- valued hesitant fuzzy ranking method based on group decision analysis for green supplier selection," IFAC-Papers OnLine, vol. 49, no. 2, pp. 12-17, 2016.

[22] W.-C. Yeh and M.-C. Chuang, "Using multi-objective genetic algorithm for partner selection in green supply chain problems," Expert Systems with Applications, vol. 38, no. 4, pp. 4244-4253, 2011.

[23] A. Kumar, V. Jain, S. Kumar, and C. Chandra, "Green supplier selection: a new genetic/immune strategy with industrial application," Enterprise Information Systems, vol. 10, no. 8, pp. 911943, 2016.

[24] M. Gupta, "Supplier selection using artificial neural network and genetic algorithm," International Journal of Indian Culture and Business Management, vol. 11, no. 4, pp. 457-472, 2015.

[25] M. Punniyamoorthy, P. Mathiyalagan, and P. Parthiban, "A strategic model using structural equation modeling and fuzzy logic in supplier selection," Expert Systems with Applications, vol. 38, no. 1, pp. 458-474, 2011.

[26] A. Fallahpour, E. U. Olugu, S. N. Musa, D. Khezrimotlagh, and K. Y. Wong, "An integrated model for green supplier selection under fuzzy environment: application of data envelopment analysis and genetic programming approach," Neural Computing and Applications, vol. 27, no. 3, pp. 707-725, 2016.

[27] L.-H. Li, J.-C. Hang, Y. Gao, and C.-Y. Mu, "Using an Integrated Group Decision Method Based on SVM, TFN-RS-AHP, and TOPSIS-CD for Cloud Service Supplier Selection," Mathematical Problems in Engineering, vol. 2017, Article ID 3143502, 14 pages, 2017.

[28] A. Amid, S. H. Ghodsypour, and C. O’Brien, "A weighted maxmin model for fuzzy multi-objective supplier selection in a supply chain," International Journal of Production Economics, vol. 131, no. 1, pp. 139-145, 2011.

[29] Z. Hu, C. Rao, Y. Zheng, and D. Huang, “Optimization decision of supplier selection in green procurement under the mode of low carbon economy," International Journal of Computational Intelligence Systems, vol. 8, no. 3, pp. 407-421, 2015.

[30] M. Tavana, A. Fallahpour, D. Di Caprio, and F. J. Santos-Arteaga, "A hybrid intelligent fuzzy predictive model with simulation for supplier evaluation and selection," Expert Systems with Applications, vol. 61, no. 11, pp. 129-144, 2016.

[31] J. Qin, X. Liu, and W. Pedrycz, "An extended TODIM multicriteria group decision making method for green supplier selection in interval type-2 fuzzy environment," European Journal of Operational Research, vol. 258, no. 4, pp. 626-638, 2017.

[32] P. Ghadimi, A. Dargi, and C. Heavey, "Sustainable supplier performance scoring using audition check-list based fuzzy inference system: a case application in automotive spare part industry," Computers and Industrial Engineering, vol. 105, pp. 12-27, 2017.

[33] Z. Xu, "Intuitionistic preference relations and their application in group decision making," Information Sciences, vol. 177, no. 11, pp. 2363-2379, 2007.

[34] A. Čižmešija, "A new sharp double inequality for generalized Heronian, harmonic and power means," Computers and Mathematics with Applications, vol. 64, no. 4, pp. 664-671, 2012.

[35] P. Liu, Z. Liu, and X. Zhang, "Some intuitionistic uncertain linguistic Heronian mean operators and their application to group decision making," Applied Mathematics and Computation, vol. 230, pp. 570-586, 2014.

[36] Z.-P. Tian, J. Wang, J.-Q. Wang, and H.-Y. Zhang, "A likelihoodbased qualitative flexible approach with hesitant fuzzy linguistic 
information," Cognitive Computation, vol. 8, no. 4, pp. 670-683, 2016.

[37] Z. S. Xu, "On multi-period multi-attribute decision making," Knowledge-Based Systems, vol. 21, no. 2, pp. 164-171, 2008.

[38] Z. S. Xu and J. Chen, "Binomial distribution based approach to deriving time series weights," in Proceedings of the 2007 IEEE International Conference on Industrial Engineering and Engineering Management, IEEM 2007, pp. 154-158, Singapore, December 2007.

[39] Z. Xu and R. R. Yager, "Dynamic intuitionistic fuzzy multiattribute decison making," International Journal of Approximate Reasoning, vol. 48, no. 1, pp. 246-262, 2008.

[40] R. Sadiq and S. Tesfamariam, "Probability density functions based weights for ordered weighted averaging (OWA) operators: an example of water quality indices," European Journal of Operational Research, vol. 182, no. 3, pp. 1350-1368, 2007.

[41] Z.-L. Yang and L.-C. Huang, "Dynamic stochastic multiattribute decision-making that considers stochastic variable variance characteristics under time-sequence contingency environments," Mathematical Problems in Engineering, vol. 2017, Article ID 7126856, 9 pages, 2017.

[42] J. Sarkis, Q. Zhu, and K.-H. Lai, "An organizational theoretic review of green supply chain management literature," International Journal of Production Economics, vol. 130, no. 1, pp. 1-15, 2011.

[43] K. Atanassov and G. Gargov, "Interval valued intuitionistic fuzzy sets," Fuzzy Sets and Systems, vol. 31, no. 3, pp. 343-349, 1989.

[44] Z.-S. Xu, "Methods for aggregating interval-valued intuitionistic fuzzy information and their application to decision making," Control and Decision, vol. 22, no. 2, pp. 215-219, 2007 (Chinese).

[45] V. L. G. Nayagam, S. Muralikrishnan, and G. Sivaraman, "Multicriteria decision-making method based on interval-valued intuitionistic fuzzy sets," Expert Systems with Applications, vol. 38, no. 3, pp. 1464-1467, 2011.

[46] R. R. Yager, "OWA aggregation over a continuous interval argument with applications to decision making," IEEE Transactions on Systems, Man, and Cybernetics, Part B: Cybernetics, vol. 34, no. 5, pp. 1952-1963, 2004.

[47] H. Zhao, J.-X. You, and H.-C. Liu, "Failure mode and effect analysis using MULTIMOORA method with continuous weighted entropy under interval-valued intuitionistic fuzzy environment," Soft Computing, vol. 10, no. 3, pp. 1-13, 2016.

[48] D. J. Yu, "Intuitionistic fuzzy geometric Heronian mean aggregation operators," Applied Soft Computing, vol. 13, no. 2, pp. 1235-1246, 2013.

[49] Z. Xu and R. R. Yager, "Some geometric aggregation operators based on intuitionistic fuzzy sets," International Journal of General Systems, vol. 35, no. 4, pp. 417-433, 2006. 


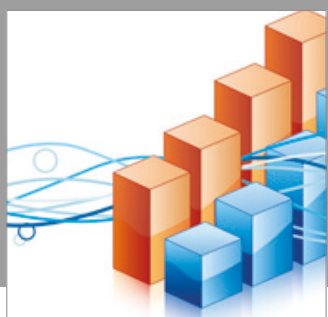

Advances in

Operations Research

vatersals

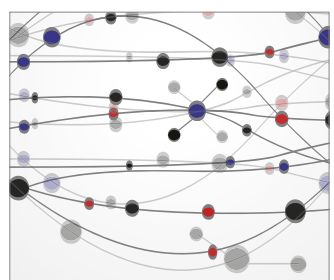

\section{The Scientific} World Journal
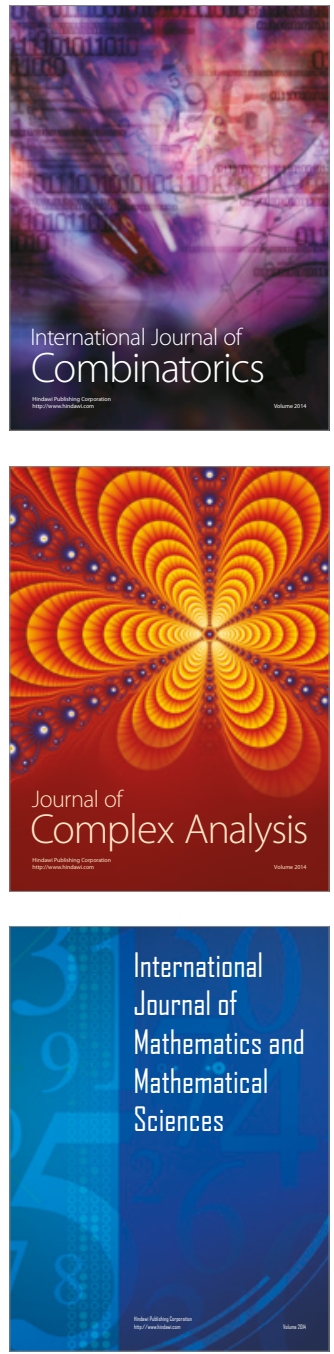
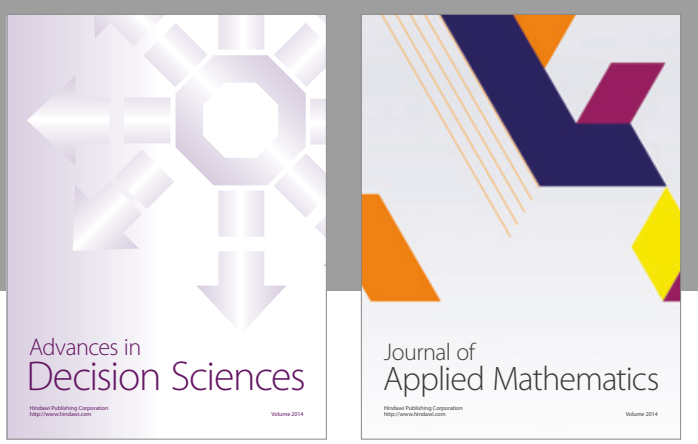

Algebra

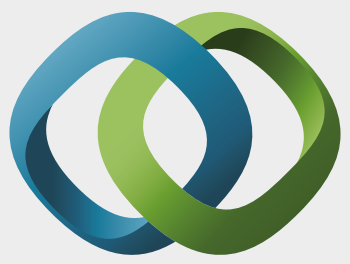

\section{Hindawi}

Submit your manuscripts at

https://www.hindawi.com
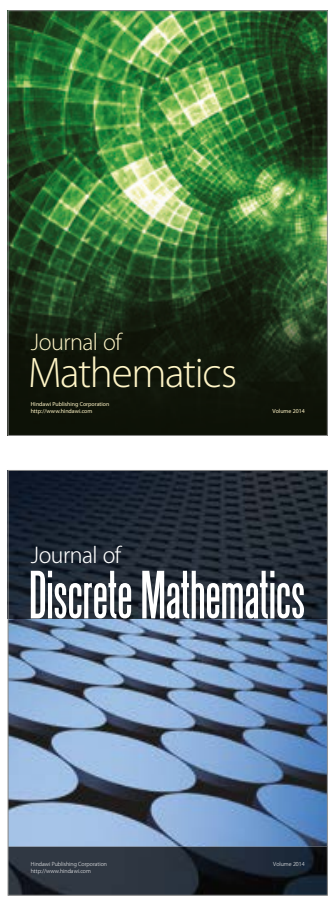

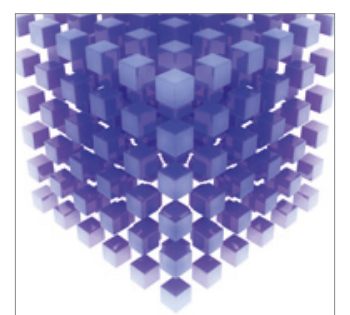

Mathematical Problems in Engineering
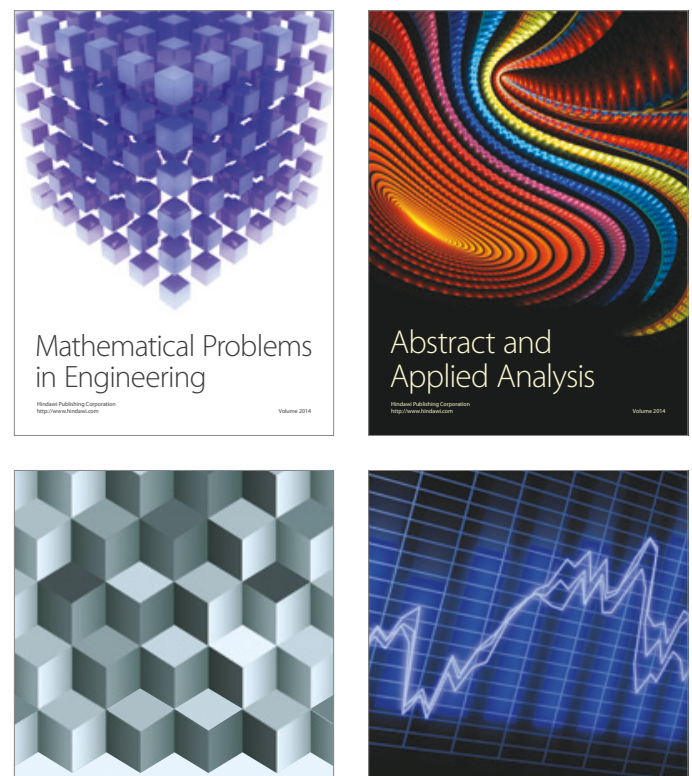

Journal of

Function Spaces

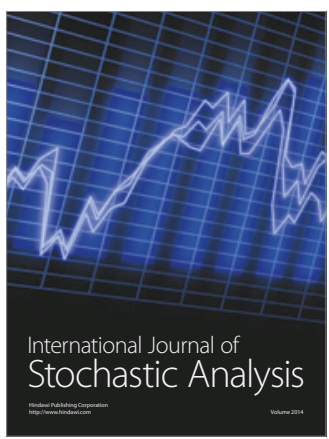

Probability and Statistics
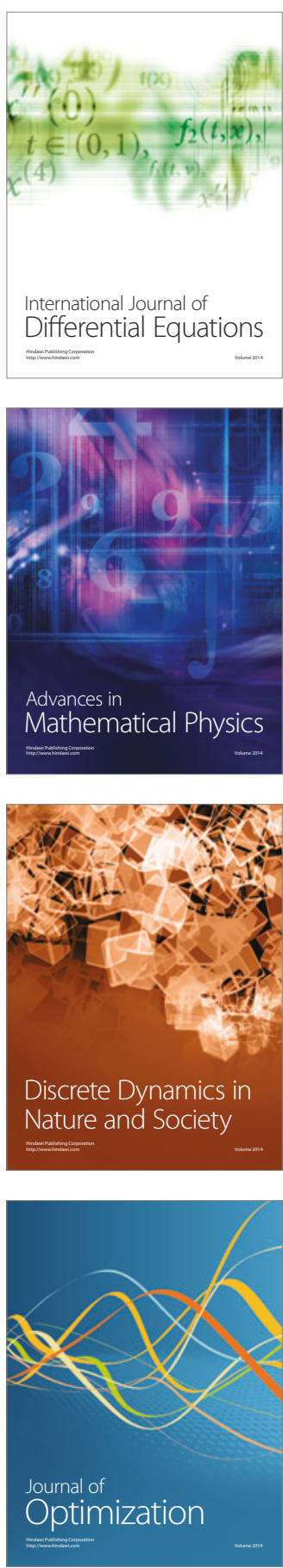